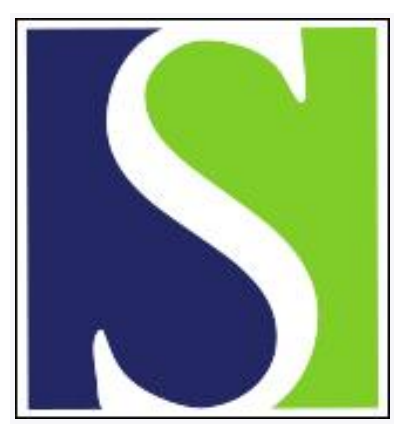

Scand J Work Environ Health 1988;14(1):31-35

https://doi.org/10.5271/sjweh.1953

Issue date: Feb 1988

Ultrastructural changes in peripheral nerves of the fingers of three vibration-exposed persons with Raynaud's phenomenon.

by Takeuchi T, Takeya M, Imanishi H

Affiliation: Department of Food and Nutrition, Shokei College, Kumamoto, Japan.

This article in PubMed: www.ncbi.nlm.nih.gov/pubmed/3353694

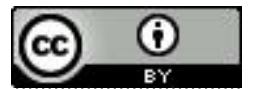




\title{
Ultrastructural changes in peripheral nerves of the fingers of three vibration-exposed persons with Raynaud's phenomenon
}

\author{
by Tadao Takeuchi, MD, ${ }^{1}$ Motohiro Takeya, MD, ${ }^{2}$ Hayao Imanishi, MD ${ }^{3}$
}

\begin{abstract}
TAKEUCHI T, TAKEYA M, IMANISHI H. Ultrastructural changes in peripheral nerves of the fingers of three vibration-exposed persons with Raynaud's phenomenon. Scand J Work Environ Health 14 (1988) 31-35. A finger biopsy was performed on three patients with vibration-induced white finger (VWF), and the specimens were examined by electron microscopy for peripheral nerve changes. A characteristic neuropathy with perineurial fibrosis was revealed which was often accompanied by a thickened perineurium with a lamellar structure resembling onion skin. This peculiar fibrosis consisted of elongated cytoplasmic projections of perineurial cells or fibroblasts and a greatly increased amount of collagen which occasionally contained fibrous long-spacing collagen. In the endoneurium, a decrease in the number of nerve fibers and a marked increase in collagen with fibroblasts were noted. Myelinated axons became smaller, and this occurrence suggested incomplete regeneration. The pathological changes were presumably the result of the long-term clinical course of VWF.
\end{abstract}

Key terms: case report, electron microscopy, light microscopy, neuropathy, perineurial fibrosis, vibration, vibration-induced white finger.

Changes in the peripheral nerves of patients with vibration-induced white finger (VWF) have been suggested from clinical and pathophysiological examinations by Lukas (8) and Torii et al (14), although Walton (15) failed to find such changes in his autopsy study. Experimentally, however, Karpova (7) recognized Wallerian degeneration in the peripheral nerves of rabbits. In their pathological examination of skin specimens from 30 patients with VWF, Takeuchi et al (11) found a clear increase in the thickness of the perineurium caused by connective tissue and marked perineurial fibrosis and muscular hypertrophy of the arterial walls, the last finding having also been described earlier by Ashe et al (1).

The objective of the present study was to determine the characteristics of the changes found in the peripheral nerves of VWF patients. For this purpose, we used electron microscopy, which allowed us to examine the pathological findings of the nerves in detail.

\section{Subjects and methods}

Finger biopsies of three patients with VWF were performed with the approval of the individuals concerned. The first patient was a 55-year-old man who had been exposed to vibration from a rammer for 12 years. He had had numbness, minor pain, and finally Raynaud's phenomenon in four fingers (II-V) of both hands for

\footnotetext{
Pathology Division, Department of Food and Nutrition, Shokei College, Kumamoto, Japan.

2 Department of Pathology, Kumamoto University Medical School, Kumamoto, Japan.

${ }^{3}$ Imanishi Hospital, Kochi, Japan.
}

Reprint requests to: Dr T Takeuchi, 6-3 Hieda-Machi, Kumamoto 860 , Japan. the last three years. The skin biopsy was performed in the region of the middle phalanx of the third finger and the distal phalanx of the fourth finger of the left hand by means of a skin-cutting procedure.

The second patient, a man 60 years of age, had used a pneumatic hammer and pick for 13 years. Thereafter, he complained of numbness and pain in his hands and fingers. Raynaud's phenomenon appeared in four fingers (II-V) of his left hand, and the attacks continued for several years after he stopped using vibrating tools. The biopsy specimen was taken from the region of the distal phalanx of the fourth finger of the left hand by the same method as was used for the first patient.

The third patient was a 68 -year-old man who had had 15 years' heavy exposure to chain saws. He started complaining of numbness and pain seven years after he first began using the saws. He had also experienced severe symptoms of Raynaud's phenomenon in all fingers of both hands. Muscle atrophy of the hand was observed at the time of the biopsy. A reduced induction velocity of the radial nerve was also found in his right hand. The biopsy was performed in the area of the middle phalanx of the third finger of the right hand and the distal phalanx of the fourth finger of the right hand with the same method used for patient 1 .

The biopsy specimens of all three patients were fixed in $2.5 \%$ glutaraldehyde and buffered with $0.1 \mathrm{M}$ sodium cacodylate and $2 \%$ sucrose at $4^{\circ} \mathrm{C}$ for one night. They were then postfixed in $1 \%$ osmium tetroxide for $1 \mathrm{~h}$. Routine embedding in epoxy resin and cutting on an ultrotome were performed. Samples of $1 \mu \mathrm{m}$ were routinely stained with toluidine blue and examined by light microscopy. Thereafter, the marked, thinly cut samples were stained with lead and observed under an electron microscope (Hitachi 12A). 


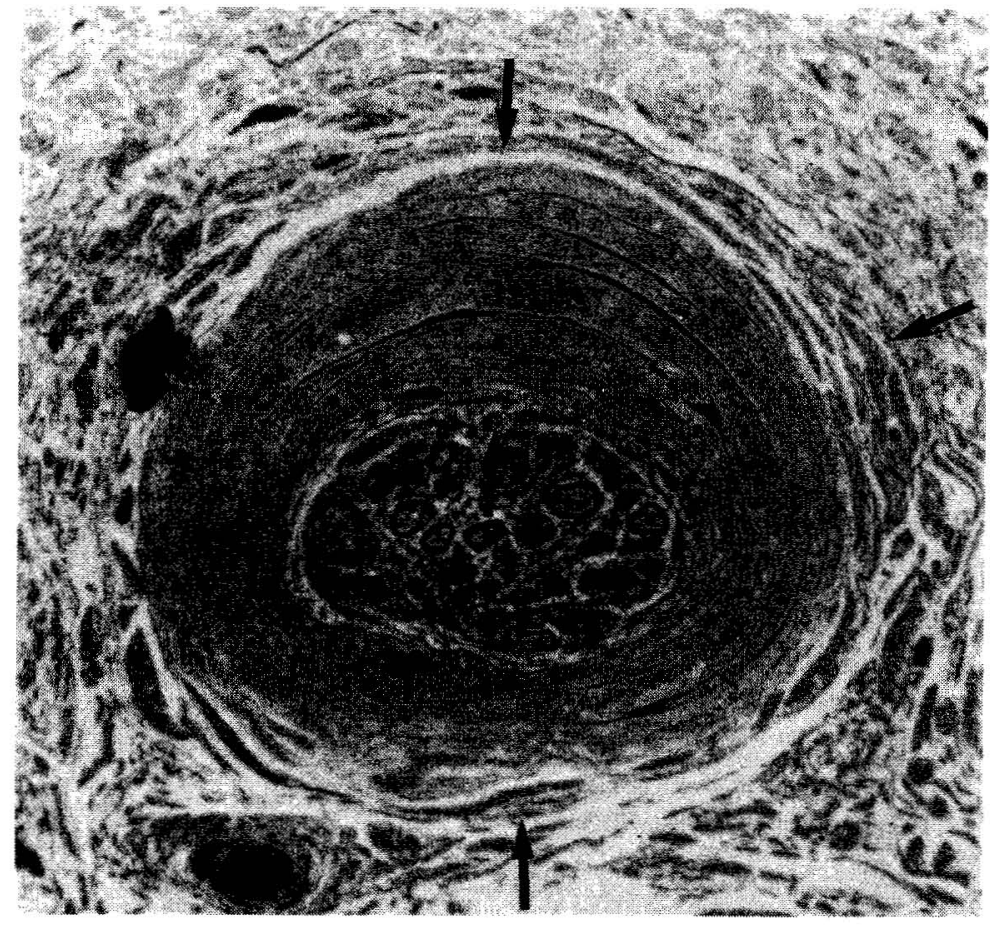

Figure 1. Light micrograph of a cross. section of a digital peripheral nerve from a 1-um thick specimen stained with toluidine blue (patient 2) showing a thickened perineurium which resembles onion skin and which is primarily the result of abundant fibrosis with elongated perineurial cells (arrows) $(580 \times)$

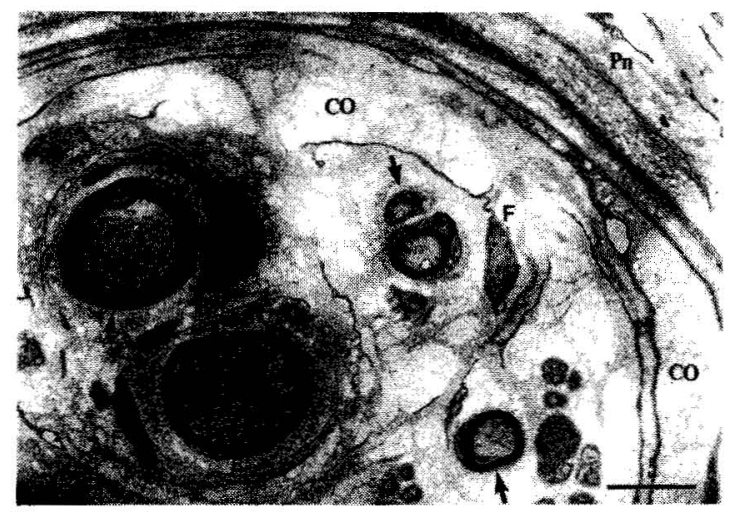

Figure 2. Electron micrograph of a digital peripheral nerve (patient 2) showing a decrease in the number of myelinated nerve fibers including small myelinated nerve fibers (arrows). Endoneurial fibrosis apparently occurred and formed larger and smaller bundles of collagen (CO) with fibroblasts. The perineurium is thickened with fibrosis. (bar $=5 \mu \mathrm{m}$ )

As new control specimens from humans could not be obtained, the specimens from five persons from a previous study (11) were used as reference also in this study.

\section{Results}

In the examination by light microscopy, perineurial fibrosis and a predominant thickening of the perineurium were noted in the specimens from all three

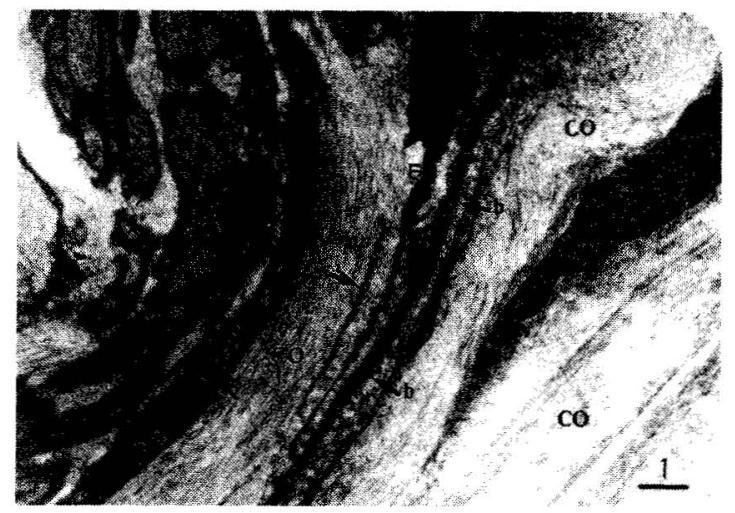

Figure 3. Electron micrograph showing perineurial fibrosis (patient 3). The thickened lamellar layers consists of increased collagen (CO) and projections of the elongated cytoplasm of increased perineurial cells with basement membrane (b) and fibroblasts. Typical fibrous long-spacing collagen (arrows) is also seen. $(\mathrm{bar}=1 \mu \mathrm{m})$

patients. The perineurium itself formed a lamellar structure resembling onion skin. This structure was often seen on the surface of the transverse section of the peripheral nerve (figure 1). Collagen tissue was increased in the elongated cytoplasm of the perineurial cells. Myelinated nerve fibers were frequently decreased in number, and small nerve fibers not usually observed under normal conditions were found. Axons were also decreased in number and were smaller in size, whereas the myelin sheath was reduced in thickness. The remaining nerve fibers were loosely distributed in the 
endoneurium, where the connective tissue was increased. Similar findings were observed for the structure of the peripheral nerves in all three individuals. On the other hand, the reference material did not show a thickening of the perineurium in any case, although the prepared slides were examined again in connection with the present study.

The electron microscopy revealed the aforementioned findings and structures even more distinctly. The thickened concentric lamellar layers of the perineurium were composed of elongated cytoplasmic projections of perineurial cells and greatly increased amounts of collagen (figures 2 and 3). The perineurial cells themselves were increased, and their cytoplasmic projections were also extremely elongated. Some of the projections in the inner layer of the perineurium protruded into the endoneurium (figures 2 and 4 ). The nuclei, intracytoplasmic vesicles, and mitochondria of these cells had remained within almost normal limits during the prolonged course of development of the revealed changes. Among the perineurial cells and projections which had a basement membrane (basal lamina), the amount of collagen was greatly increased and contained fibroblasts without a basement membrane. The collagen bundles sometimes contained the so-called long-spacing collagen described by Luse (9), and they varied in size and length (figure 3 ).

Nerve fibers were decreased in number to a considerable extent in the endoneurium, which had become loose and edematous as a result (figures 2 and 5). Axons and myelinated sheaths were also decreased in diameter and thickness and therefore suggested incomplete regeneration of nerve fibers following damage. The small size observed was usually not seen in the reference material. In addition the endoneurium was infiltrated with collagen containing fibroblasts (figure 5).

Axons in the myelinated sheaths were smaller and more slender (figures 4 and 5). They were distributed irregularly and were loosely scattered (figures 1, 2, and $5)$. The number of mitochondria varied and was sometimes increased (figure 6), but there were no specific changes in the fine structures, except for the smaller size. Vesicles of irregular size and form occasionally occurred in the submyelinated area and in the matrix of the axons (figures 2 and 5). No unusual characteristics or structural changes were found in the individual neurofilaments and neurotubules.

Myelin sheaths were frequently changed in the thinner layer, and sometimes the cleft appeared to occur once or, rarely, twice between layers of lamellar myelin (figures 2 and 5). There was an occasional separation of lamellae near the Schmidt-Lantermann cleft, as well as marked changes in the cleft, with myelin bodies of different sizes and forms in the matrix.

Schwann cells frequently contained a smaller myelinated axon that varied in size in individual cases; this axon had possibly been regenerated and sometimes ap-

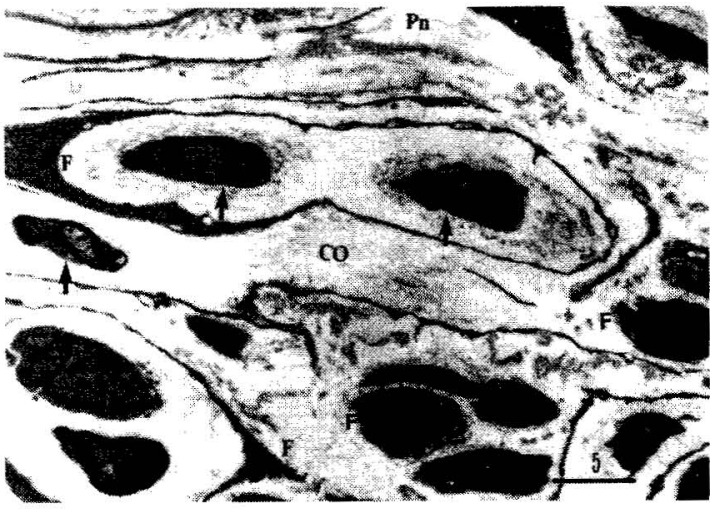

Figure 4. Electron micrograph of moderate fibrosis in a digita peripheral nerve (patient 3). Moderately increased collagen (CO) occurs in both the perineurium (Pn) and endoneurium, and the number of myelinated nerve fibers (arrow) is decreased. $(\mathrm{bar}=5 \mu \mathrm{m})$

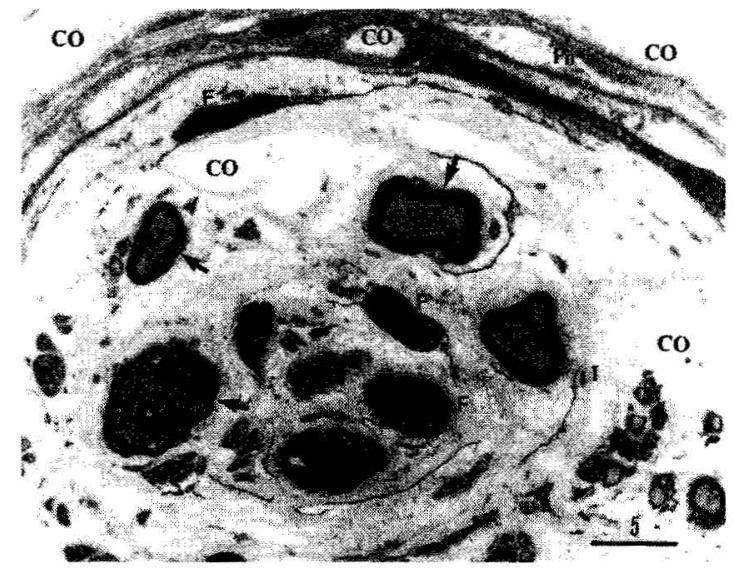

Figure 5. Electron micrograph of marked neuropathy of a digital peripheral nerve (patient 3) showing a decrease in the number of myelinated nerve fibers (arrows). Incomplete regeneration of nerve fibers and marked fibrosis $(\mathrm{CO})$ with fibroblasts (F) can be seen in the endoneurium. Smaller axons are also present. (bar $=5 \mu \mathrm{m}$ )

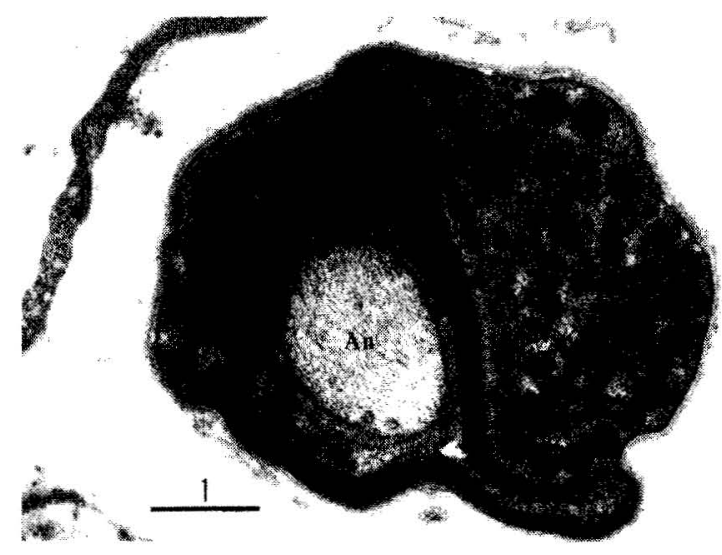

Figure 6. Electron micrograph of an active Schwann cell including a regenerated axon (An) with incomplete myelination. Mitochondria are increased in number. (bar $=1 \mu \mathrm{m}$ ) 
peared as extremely fine in the cytoplasmic matrix near the nucleus (figures 4 and 5). Mitochondria were often increased in number in the cytoplasm and sometimes their structure was elongated (figure 6). The glycogen particles were apparently increased in number, and some of them were also increased in size, forming considerably larger particles of rosettes, described as $\boldsymbol{\beta}$ particles by Drochmans (2).

The unmyelinated fibers were also decreased in number and size and were dispersed (figure 5). This finding suggests possible regeneration after injury. Extremely fine axons without myelin were sometimes observed in the cytoplasm near the nucleus of the Schwann cells (figure 2).

In the endoneurium, bundles of collagen varying in size were loosely scattered, and homogeneous fluid suggesting edema filled the space between the collagen bundles and nerve fibers (figures 2 and 5). The collagen bundles were also increased in number and were accompanied by newly formed fibroblasts (figures 4 and 5). Fibrous long-spacing collagen rarely occurred in the endoneurium. Similar findings were also observed around the thickened perineurium.

The changes found in patients 2 and 3 proved to be particularly marked.

\section{Discussion}

It is well known that Raynaud's phenomenon in association with occupational exposure to vibration is closely related to intense contraction of the local arteries, in which medial muscular hypertrophy occurs with an increase in the thickness of the arterial walls $(1,11)$. On the other hand, the clinical signs and symptoms of VWF indicate the presence of polyneuropathy $(8,14)$, and Karpova $(7)$ also suggested its occurrence from experimental data on rabbits. In addition Takeuchi et al (11) took biopsy specimens from the fingers of 30 patients with VWF and found that the peripheral nerves were always damaged and that severe changes were evident.

In the present study nerve changes similar to those observed previously were confirmed for all three patients in the light microscopic examination. The findings indicated a decrease in the number of myelinated nerve fibers and a characteristic appearance of perineurial lamellar thickness which was not observed in the reference material (11).

Under the electron microscope the first notable feature was thick lamellar fibrosis of the perineurium which resembled onion skin in transverse sections. The thick perineurium consisted of elongated cytoplasmic projections of increased perineurial cells with a greatly increased amount of collagen among them. Such thick perineurial fibrosis has not been reported in other forms of polyneuropathy described in the literature (3, $5,6,10,12,13)$. The amount of collagen was also increased around the perineurium itself, and this finding also indicated perineurial fibrosis. Such a marked in- crease in collagen presumably resulted from the longterm presence of repeated vibration and the separation of the nerve bundles from the surrounding tissue as a result of exposure to intense vibration. A similar mechanism possibly operated around the artery and led to the thick periarterial fibrosis observed in one of our previous studies (11).

Another characteristic determined with the aid of electron microscopy was a decrease in the number of nerve fibers. The nerve fibers were often extremely small in size, and the myelinated axons were also smaller, presumably suggesting some regeneration similar to that which occurs in the polyneuropathy related to Minamata disease (4). The Schwann cells were increased in number and were often rich in mitochondria, which were probably active in the regeneration of myelinated nerve fibers with thinner myelin sheaths. Therefore, the Schwann cells often contained a smaller or fine axon within their cytoplasm. The decrease in the number of nerve fibers resulted in an increased amount of collagen with fibroblasts in the endoneurium, and it also caused the perineurial cells to project into the endoneurium. The borderline areas between the perineurium and endoneurium therefore became unclear and broader and were infiltrated by fibroblasts. The long-term clinical course of the VWF presumably led to these pathological changes.

The aforementioned findings suggest that repeated exposure to intense vibration may produce a characteristic nerve disturbance which is somewhat different from toxic or metabolic neuropathies, although it remains to be confirmed whether other physical mechanisms may produce a similar fibrous thickening of the perineurium.

\section{References}

1. Ashe WF, Cook WT, Old JW. Raynaud's phenomenon of occupational origin. Arch Environ Health 5 (1962) $333--343$

2. Drochmans P. Morphologie de glycogene. J Ultrastruct Res 6 (1962) 141-163.

3. Dyck PJ, Thomas PK, Lambert EH, ed. Peripheral neuropathy. Volumes I and II. Saunders Co, Philadelphia, London, Toronto 1975.

4. Eto K, Takeuchi T. Pathological changes of human sural nerves in Minamata disease (methylmercury poisoning): Light and electron microscopic studies. Virchows Arch B 23 (1977) 109-128.

5. Goldstein NP, McCall JT, Dyck PJ. Metal neuropathy. In: Dyck PJ, Thomas PK, Lambert EH, ed. Peripheral neuropathy. Volume II. Saunders Co, Philadelphia, London, Toronto 1975, pp 1227-1262.

6. Hopkins A. Toxic neuropathy due to industrial agents. In: Dyck PJ, Thomas PK, Lambert EH, ed. Peripheral neuropathy. Volume II. Saunders Co, Philadelphia, London, Toronto 1975, pp 1207-1226.

7. Karpova NI. Vibration and nervous system. Leningrad Medicine, Leningrad 1976, pp 66-147.

8. Lukas E. Peripheral nervous system and hand-arm vibration exposure. In: Brammer AJ, Taylor W, ed. The vibration effects on the hand and arm in industry. John Wiley \& Sons, New York, NY 1982, pp 39-44. 
9. Luse SA. Electron microscopic studies of brain tumors. Neurology $10(1960) 881-905$.

10. Luse SA, Moon TR. Ultrastructural siudy of peripheral nerves in human diabetes mellitus and in steroid-induced hyperglycemia in the rabbit. Anat Rec 154 (1966) 380395.

11. Takeuchi T, Futatsuka $\mathbf{M}$, Imanishi $\mathbf{H}$, Yamada $\mathrm{S}$. Pathological changes observed in the finger biopsy of patients with vibration-induced white finger. Scand $\mathrm{J}$ Work Environ Health 12 (1986) 280-283.

12. Thomas PK, Eliasson SG. Diabetic neuropathy. In: Dyck PJ, Thomas PK, Lambert EH, ed. Peripheral neuropathy. Volume II. Saunders Co, Philadelphia, London, Toronto 1975, pp 956-981.

13. Thomas PK, Olsson Y. Microscopic anatomy and func- tion of the connective tissue components of peripheral nerve. In: Dyck PJ, Thomas PK, Lambert EH, ed. Peripheral neuropathy. Volume I. Saunders Co, Philadelphia, London, Toronto 1975, pp 168-189.

14. Torii J, Kohsaka K, Imanishi H. Disturbance of peripheral nerves by use of chain saw: Observation of motor nerve conduction velocity [in Japanese]. Nippon Iji Shinpo 2829 (1978) 32-34.

15. Walton $\mathrm{KW}$. The pathology of Raynaud's phenomenon of occupational origin. In: Taylor W, ed. The vibration syndrome. Academic Press Inc, London 1974, pp 109 120.

Received for publicatıon: 30 March 1987 